Bull. Mater. Sci., Vol. 6, No. 3, July 1984, pp. 503-512. (C) Printed in India.

\title{
X-ray microanalysis in the transmission electron microscope
}

\author{
G W LORIMER
}

Joint University of Manchester/UMIST, Department of Metallurgy, Grosvenor Street, Manchester, M1 7HS, England.

\begin{abstract}
The technique of x-ray microanalysis of thin specimens in the transmission electron microscope is well established. Quantitative analyses can be obtained by converting observed $\mathrm{x}$-ray intensity ratios into weight fraction ratios by incorporating suitable correction factors.

Flourescence corrections in thin specimens are always significantly less than in bulk specimens and, except where strong characteristic radiation fluorescence enhancement is predicted in the bulk, can be safely ignored. As analysis probes less than $100 \AA$ diameter have become available, the beam spreading has become an important parameter in determining the spatial resolution for analysis.

Present developments are directed at improving the quality of the analyses which can be obtained. This requires the generation of high quality correction factors, particularly for $Z<13$, rapid and accurate methods of determining sample thickness, so that absorption corrections can be made, and the development of reliable procedures for determining the shape of the activated volume in a thin specimen.
\end{abstract}

Keywords. X-ray microanalysis; transmission electron microscopy; quantitative model: fluorescence and absorption corrections; intensity ratio; beam broadening.

\section{Introduction}

In a thin specimen the spatial resolution for chemical analysis is dramatically improved, compared to the bulk; the large, bell-shaped region produced by the diffusion of the electron probe beneath the surface of the specimen, from which majority of the $x$-rays are produced in a bulk sample, is absent in a thin foil (figure 1). If the sample is sufficiently thin to carry out quantitative transmission electron microscopy at $100 \mathrm{kV}$ the activated volume is approximately a cylinder equal to the beam diameter.

\section{Quantitative analysis: thin film approximation}

Characteristic $\mathrm{x}$-ray fluorescence and $\mathrm{x}$-ray absorption in thin specimens is less than in bulk samples (figure 1). To a first approximation $x$-ray absorption and fluorescence can be neglected and the ratio of two observed $\mathrm{x}$-ray intensities, $I_{A} / I_{B}$, can be related to the corresponding weight-fraction ratio, $C_{A} / C_{B}$, by the equation

$$
C_{A} / C_{B}=k_{A B}\left(I_{A} / I_{B}\right) \text {, }
$$

where $k_{A B}$ is a constant at a given accelerating voltage and is independent of specimen thickness and composition. $k_{A B}$ values can be determined experimentally (Cliff and Lorimer 1975) or calculated (Goldstein et al 1977), by

$$
k_{X \mathrm{Si}}=\frac{A_{X}\left(Q_{K} \omega_{K} a\right)_{\mathrm{Si}} \exp \left(-\mu_{\mathrm{Be}}^{\mathrm{Si}} \rho t\right)}{A_{\mathrm{Si}}\left(Q_{K} \omega_{K} a\right)_{X} \exp \left(-\mu_{\mathrm{Be}}^{X} \rho t\right)},
$$


where $A_{X}$ and $A_{\mathrm{Si}}$ are atomic weights, $Q_{K}$ the Green and Cosslett (1961) values of the $K$ shell ionization cross-sections, $\omega_{K}$ the fluorescence yield, $a$ the $K_{\alpha} /\left(K_{\alpha}+K_{\beta}\right)$ ratio (equal to one for $Z<19$ ) and $\mu_{\mathrm{Bc}}^{X}$ and $\mu_{\mathrm{Be}}^{\mathrm{Si}}$ the 'effective' mass-absorption coefficients of $\mathrm{Si}$ and the element $X$ in the detector window (the Be window, Si dead layer and $\mathrm{Au}$ contact film) of density $\rho$ and thickness $t$. The ionization cross-section was assumed to be constant along the electron trajectory. A normalisation procedure, e.g. $\Sigma C_{n}=1$, must be used to convert the ratios of the weight fractions into weight percentages. In some specimens assumptions must also be made concerning oxidation states: $e . g$. it is impossible to differentiate between $\mathrm{Fe}_{3} \mathrm{O}_{4}$ and $\mathrm{Fe}_{2} \mathrm{O}_{3}$ if ratios are measured, and oxygen cannot be detected.

The agreement between the experimental and calculated $k_{A B}$ values is satisfactory for $Z>\mathrm{Si}$, (figure 2), and for these elements it would appear to be sufficient merely to

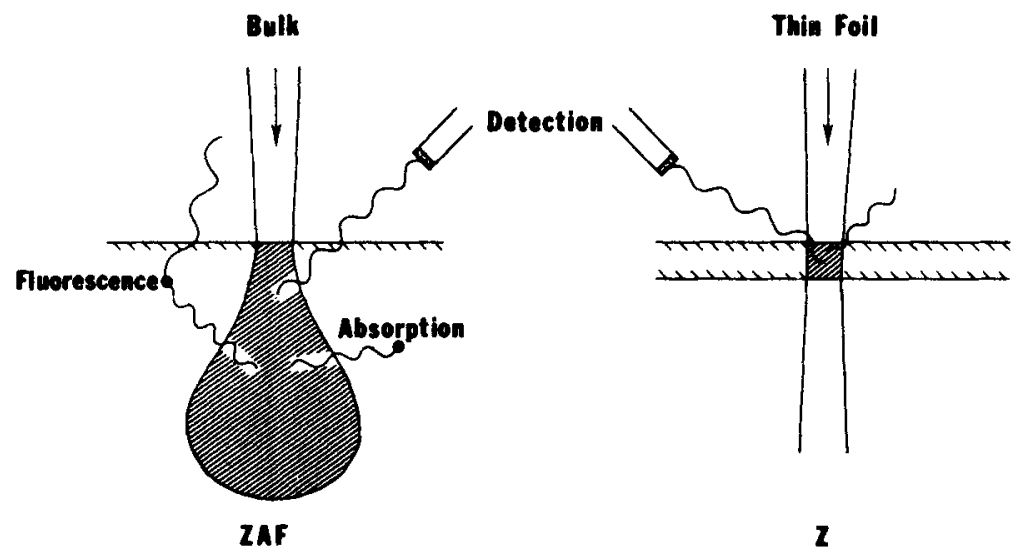

Figure 1. Schematic representation of the interaction of a high-energy electron beam with a bulk and thin-specimen. To a first approximation absorption and fluorescence effects can be ignored in the thin specimen.

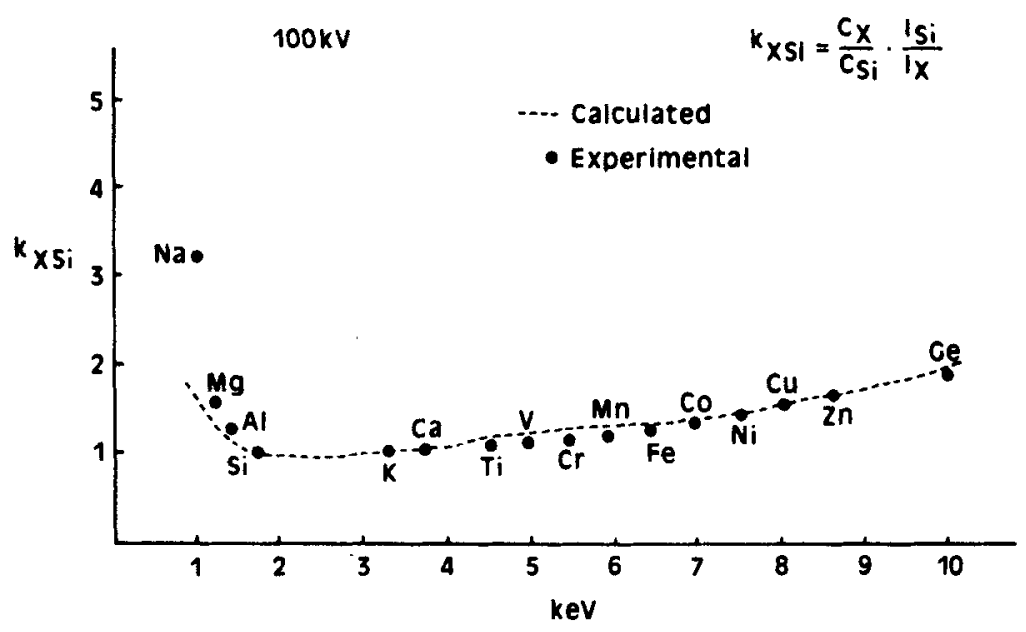

Figure 2. Comparison of calculated, Goldstein et al (1977), and experimental, Cliff and Lorimer (1975), $k_{X S i}$ values at $100 \mathrm{kV}$. 
calculate $k_{A B}$ values for a given detector and accelerating voltage. The origin of the divergence between experiment and theory at low $Z$ is not clear, but for elements $\mathrm{Na} \rightarrow \mathrm{Si}$ theory is particularly sensitive to parameters such as fluorescence yield $\left(\omega_{K}\right)$, Be window thickness etc., which are only approximately known. Another problem encountered is the loss of light elements during electron irradiation in the microscope. Figure 3 is from the work of McGill and Hubbard (1981) and shows the variation in the apparent $k_{\mathrm{NaSi}}$ value for a thin specimen of feldspar as a function of irradiation time.

Tables 1 and 2 are compiled from the experimental work of Wood et al (1981) and McGill and Hubbard (1981). They contain as good quality $k_{X \mathrm{Si}}$ values as are currently available. The McGill and Hubbard values have been extrapolated to zero time.

\section{X-ray absorption in the specimen}

The most important modification to the "thin film approximation" is where $x$-ray absorption follows the exponential law

$$
I=I_{0} \exp (-\mu \sigma x)
$$

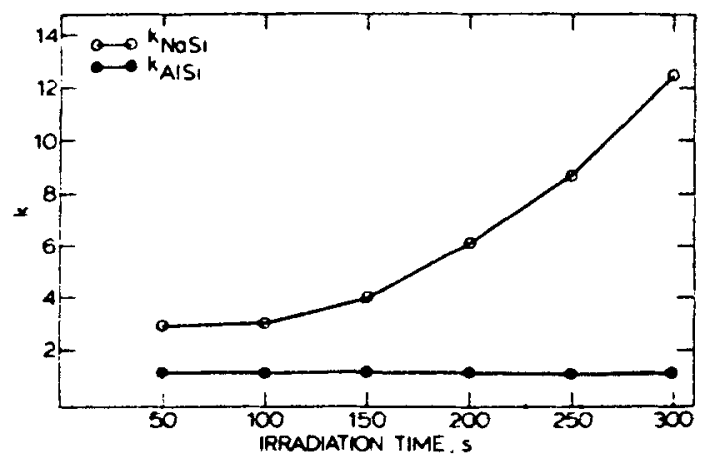

Figure 3. Variation in $k_{\mathrm{NaS}}$ and $k_{\mathrm{AISi}}$ with irradiation time for a feldspar (Amelia Albite). AEI EM6G, $100 \mathrm{kV}$, beam current $200 \mu \mathrm{A}$, beam diameter $3 \mu \mathrm{m}$. From McGill and Hubbard (1981).

Table 1. Comparison of experimental, I and calculated, $I I, k_{A S i}$ data at $120 \mathrm{kV}$.

\begin{tabular}{lccclccc}
\hline Element & Z & I & II & Element & Z & I & II \\
\hline $\mathrm{Na}$ & 11 & 3.57 & 1.83 & $\mathrm{Ti}$ & 22 & 1.12 & 1.17 \\
$\mathbf{M g}$ & 12 & 1.49 & 1.31 & $\mathrm{Cr}$ & 24 & 1.46 & 1.24 \\
$\mathrm{Al}$ & 13 & 1.12 & 1.14 & $\mathrm{Mn}$ & 25 & 1.34 & 1.32 \\
$\mathrm{Si}$ & 14 & 1.00 & 1.00 & $\mathrm{Fe}$ & 26 & 1.30 & 1.36 \\
$\mathrm{P}$ & 15 & 0.99 & 1.04 & $\mathrm{Ni}$ & 28 & 1.67 & 1.48 \\
$\mathrm{~S}$ & 16 & 1.08 & 1.07 & $\mathrm{Ca}$ & 29 & 1.59 & 1.65 \\
$\mathrm{~K}$ & 19 & 1.12 & 1.05 & $\mathrm{Mo}$ & 42 & 4.95 & 4.68 \\
$\mathrm{Ca}$ & 20 & 1.15 & 1.04 & $\mathrm{Ag}$ & 47 & 12.4 & 7.08 \\
\hline
\end{tabular}

Calculated values assume $t_{\mathrm{Be}}=7.5 \mu \mathrm{m}, t_{\mathrm{Au}}=0.02 \mu \mathrm{m}$ and $t_{\mathrm{Si}}=0.1 \mu \mathrm{m}$. From Wood et al (1981). 
where $I$ is the transmitted $\mathrm{x}$-ray intensity, $I_{0}$ the initial x-ray intensity, $\mu$ the massabsorption coefficient of a material of density $\rho$ and path length $x$. When the ratio of characteristic $\mathrm{x}$-ray intensities is considered, the difference in $\mathrm{x}$-ray absorption coefficients is the important parameter. If two characteristic $x$-rays have similar values of $\mu$ they will be absorbed equally and their intensity ratio will be unaffected.

If $t$ is the sample thickness and $\alpha$ the angle between the line of the detector and the sample surface, the x-ray intensity ratio $I_{A} / I_{B}$ recorded by the detector will be modified by absorption in the specimen from the ratio recorded for an infinitely thin specimen, $I_{0_{A}} / I_{0_{B}}$ (no absorption), as given in (4). The $x$-ray path length $x$ is shown in figure 4 .

$$
\frac{I_{A}}{I_{B}}=\frac{I_{0_{A}}}{I_{0_{B}}} \frac{\mu_{B}}{\mu_{A}} \frac{\left[1-\exp \left(-\mu_{A} \rho t \csc \alpha\right)\right]}{\left[1-\exp \left(-\mu_{B} \rho t \csc \alpha\right)\right]},
$$

where $\mu_{A}$ and $\mu_{B}$ are the mass absorption coefficients of the characteristic radiation in the sample of density $\rho$. The predicted effect of absorption in the specimen on the $x$-ray intensity ratios for orthopyroxene, a silicate mineral, is shown in figure 5.

In order to make an absorption correction it is necessary to know the x-ray path length within the specimen. There are various methods of determining the thickness of a sample, but it may be difficult to determine the $x$-ray path length with any degree of accuracy. This is particularly true for electrolytically or ion-beam-thinned samples which can be irregularly wedge-shaped. This problem has been discussed in detail by Goldstein and Williams (1981) who illustrated the large errors which could arise during the analysis of a $\mathrm{NiAl}$ sample if the shape of the foil and its orientation relative to the $\mathrm{x}$-ray detector were not known (figure 6).

Table 2. Comparison of experimental and calculated $k_{A S i}$ data at $100 \mathrm{kV}$.

\begin{tabular}{lcccc}
\hline Element & Z & I & II & III \\
\hline $\mathrm{Na}$ & 11 & 3.2 & 2.85 & 2.02 \\
$\mathbf{M g}$ & 12 & 1.6 & 1.67 & 1.39 \\
$\mathrm{Al}$ & 13 & 1.2 & 1.15 & 1.16 \\
$\mathrm{Si}$ & 14 & 1.0 & 1.00 & 1.00 \\
$\mathrm{~K}$ & 19 & 1.0 & 1.10 & 1.08 \\
$\mathrm{Ca}$ & 20 & 1.0 & 1.08 & 1.11 \\
\hline
\end{tabular}

I. Cliff and Lorimer (1975); II. McGill and Hubbard (1981); III. Calculated values. These assume $t_{\mathrm{Be}}=8 \mu \mathrm{m}, t_{\mathrm{AU}}=0.02 \mu \mathrm{m}$ and $t_{\mathrm{Si}}=0.1 \mu \mathrm{m}$.

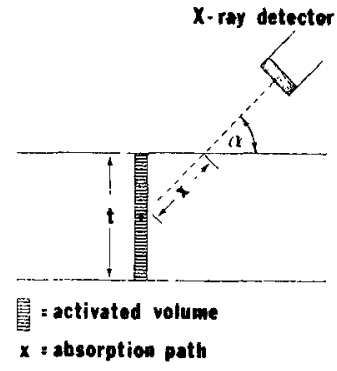

Figare 4. Geometry used for the calculation of absorption in the specimen. $X$ is the absorption path length. 


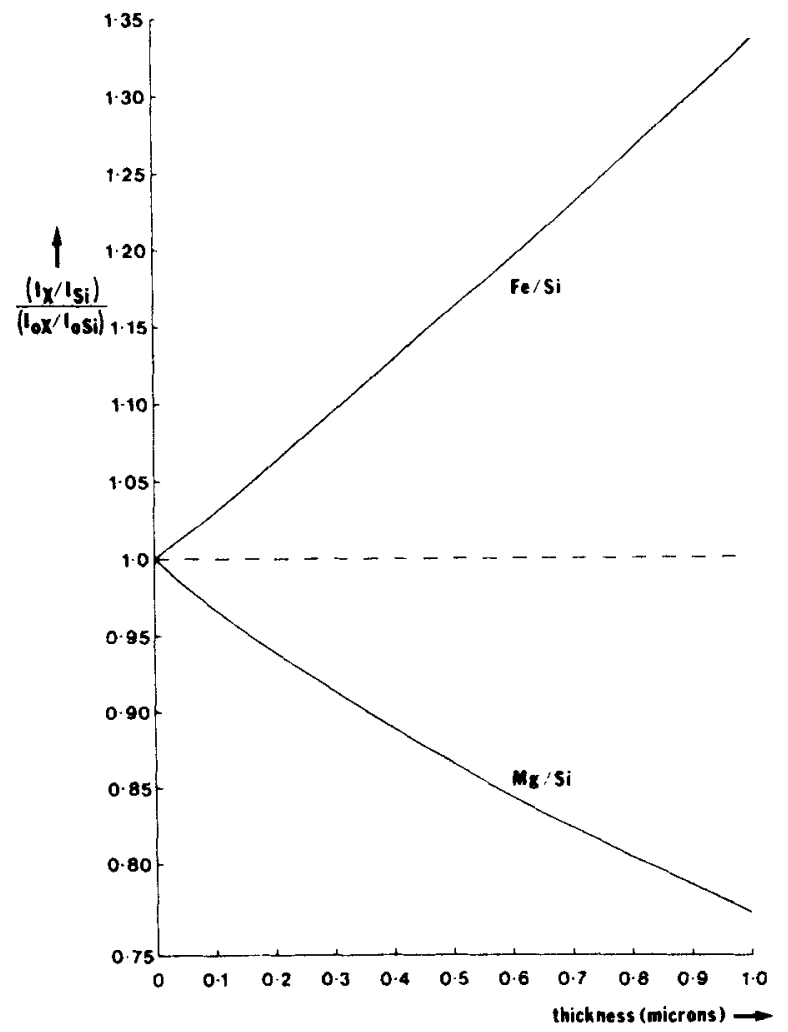

Figure 5. Effect of specimen thickness on x-ray intensity ratios for an orthopyroxene, $\mathrm{MgFeSi}_{2} \mathrm{O}_{6}$.

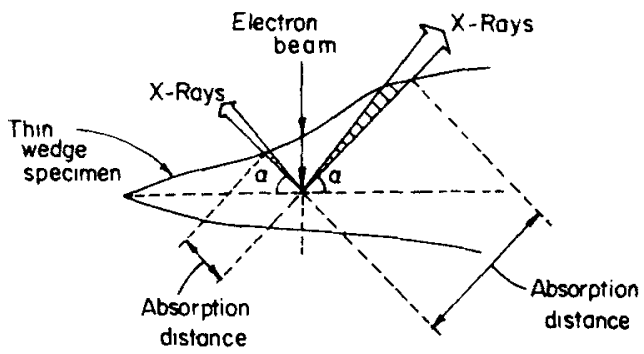

Figure 6. Possible absorption path lengths, depending on the position of the $x$-ray detector. From Goldstein and Williams (1981).

Figure 7 shows the observed variation in $\ln \left(I_{\mathrm{SiK}} / I_{\mathrm{FeK} \alpha}\right)$ as a function of specimen thickness for a $2 \mathrm{wt} \% \mathrm{Si}$ eutectoid steel which had been quenched to produce a martensitic microstructure of uniform composition. The line has a slope, as determined from least squares analysis, equal to $2090 \mathrm{~cm}^{2} \mathrm{~g}^{-1}$. This is the approximate difference between the $\mathrm{x}$-ray mass-absorption coefficients of iron $\left(2502 \mathrm{~cm}^{2} \mathrm{~g}^{-1}\right)$ and silicon $\left(710 \mathrm{~cm}^{2} \mathrm{~g}^{-1}\right) \mathrm{x}$-rays in the specimen, a value that is in agreement with that predicted in (4). 


\section{Effects of fluorescence in the specimen}

Fluorescence due to the continuum, generally ignored in bulk samples, can be similarly ignored in thin foils, moreover it is usually safe to assume that characteristic fluorescence is also very small. However, when strong characteristic fluorescence is predicted in an equivalent bulk specimen, it is necessary to account for it in the thin specimen as well. Fluorescence in thin specimens has been discussed by Philibert and Tixier (1975), Lorimer et al (1977) and Nockolds et al (1979).

The model used by Nockolds et al (1979) for calculating the fluorescence correction is shown in figure 8 . This predicts that in a thin, binary specimen $A B$ the intensity of $A$ radiation $\mathrm{d} I A$ fluoresced in the annulus by $B$ radiation from point $P$ is given by

$$
\mathrm{d} I_{F}^{A}=\frac{I_{B}}{T} C_{A} \mu_{B}^{A} \frac{r_{A}-1}{r_{A}} \omega_{K_{A}} \exp \left(-\mu_{B} \sec \phi \rho z\right) \tan \phi \mathrm{d} s \mathrm{~d} \rho z \mathrm{~d} \phi .
$$

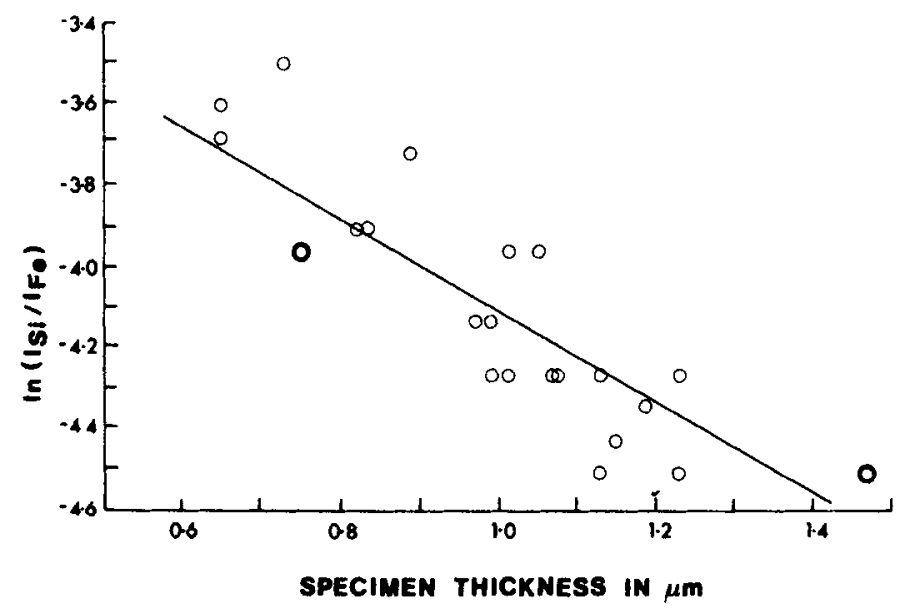

Figure 7. $\ln \left(I_{\mathrm{SiX}} / I_{\mathrm{FeKa}}\right)$ vs thickness for a $2 \mathrm{wt} \%$ Si steel. From Lorimer et al (1977).

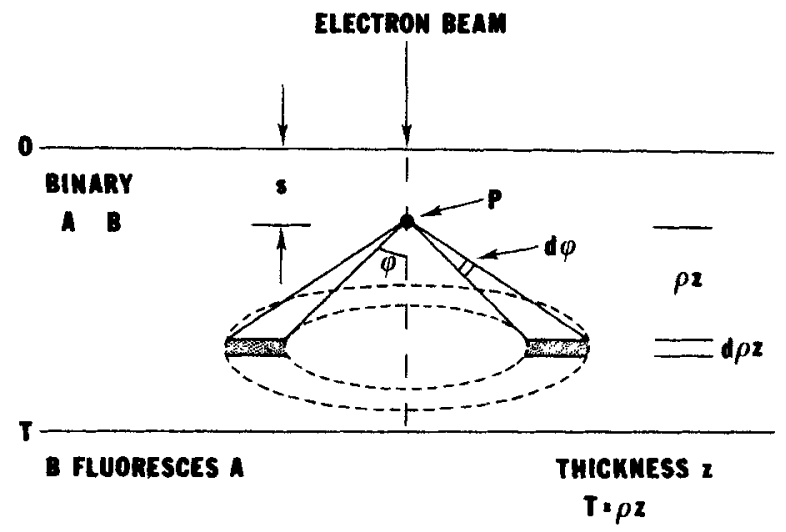

Figure 8. Model used for fluorescence correction. From Nockolds et al (1979). 
Assuming symmetry about the centre of the foil, integration over the mass thickness $T\left(\mathrm{~g} \mathrm{~cm}^{-2}\right)$ gives the total intensity $I_{F}^{A}$

$$
I_{F}^{A}=I_{B} \frac{T}{2}\left|0.923-\ln \left(\mu_{B} T\right)\right| C_{A} \mu_{B}^{A} \omega_{K_{A}} \frac{r_{A}-1}{r_{A}},
$$

$\mu_{B}^{A}$ and $\mu_{B}$ are the mass absorption coefficients of $X$-radiation from element $B$ in element $A$ and the specimen, respectively. $C_{A}$ is the weight fraction of element $A, \omega_{K_{A}}$ is the fluorescence yield of element $A$ and $r_{A}$ is the absorption-edge jump-ratio. The ratio of characteristic x-ray intensities $I_{A}$ and $I_{B}$ can be calculated using the formula for K-shell ionisation cross-sections proposed by Green and Coslett (1961), and used to modify (6) to give the fluorescence enhancement ratio for $A$ radiation $I_{F}^{A} / I_{A}$ in a thin foil

$$
\frac{I_{F}^{A}}{I_{A}}=C_{B} \cdot \omega_{K_{B}} \cdot \frac{r_{A}-1}{r_{A}} \cdot \frac{A_{A}}{A_{B}} \cdot \mu_{B}^{A} \cdot \frac{U_{B} \ln U_{B}}{U_{A} \ln U_{A}} \cdot \frac{T}{2}\left|0 \cdot 923-\ln \left(\mu_{B} T\right)\right|,
$$

where $U_{A}$ and $U_{B}$ are the overvoltage ratios for elements $A$ and $B$ respectively, and $A_{A}$ and $A_{B}$ are the relevant atomic weights. (Note that if the sample is tilted through an angle $\alpha$ then expression (7) must be multiplied by $\sec \alpha$ to allow for the resultant increase in $I_{B}$ ).

It is possible, using (7), to evaluate the fluorescence contribution from many fluorescing elements in the specimen. Thus by summing the individual contributions the total fluorescence enhancement of a particular element can be calculated and the necessary correction can be made.

\section{Surface films}

The work of Morris et al (1977) and others on aluminium alloys has clearly shown that during electropolishing solute-rich layers can be formed on the surface of the specimen, and that these will produce a marked change in the $I_{X} / I_{A l}$ ratio near the edge of the specimen. Similar effects have been observed also on electropolished samples of steels. Figure 9 shows the effect in a thin martensitic specimen of $2 \mathrm{wt} \% \mathrm{Si}$ eutectoid steel. The

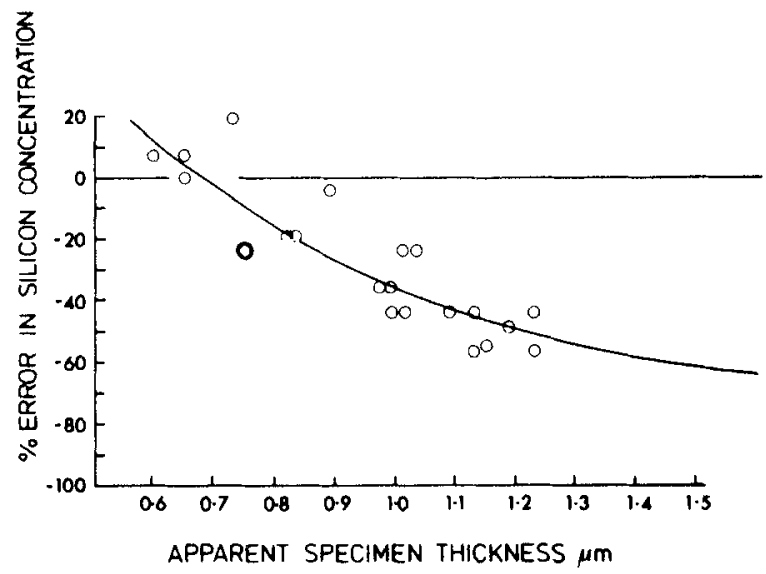

Figure 9. Error in measured concentration of silicon as function of specimen thickness for martensitic foil of $2 \mathrm{wt} \% \mathrm{Si}$ eutectoid steel. The apparent increase in Si concentration at edge of foil is due to presence of Si-rich surface film. From Ridley and Lorimer (1981). 
apparent silicon concentration is less than $2 \%$ in the thicker regions of the foil, due to the preferential absorption of $\mathrm{Si} \mathrm{K}$ radiation (discussed above) but near the edge of the sample the effect is reversed: i.e. the apparent silicon content is greater than $2 \mathrm{wt} \%$.

In this and other steel specimens it has been found that the solute-rich layer can be removed by exposing the electropolished specimen to a 10-15 min treatment in an ion beam thinning apparatus $\left(4 \mathrm{kV}, 20\right.$ to $25^{\circ}$ inclination).

\section{Beam broadening in the specimen}

Beam spreading in thin foils is currently a lively topic of both experimental investigation and theoretical analysis (refer Goldstein and Williams 1981; Doig et al 1981; Cliff and Lorimer 1981 which summarize the present state-of-the art).

A simple approach to the problem of beam spreading has been proposed by Goldstein et al (1977) who assumed that a single, elastic scattering event occurred at the centre of the foil and defined the $x$-ray source size as that volume in which $90 \%$ of the electron trajectories lie. The beam broadening $b$, from a point probe is given by:

$$
b=6.25 \times 10^{5} \frac{Z}{E_{0}}\left(\frac{\rho}{A}\right)^{1 / 2} t^{3 / 2} \mathrm{~cm},
$$

where $Z$ is the atomic number, $E_{0}$ the accelerating voltage, $\rho$ the density, $A$ the atomic weight and $t$ the sample thickness. Equation (8) was derived for a single scattering event, and hence is strictly valid for only very thin foils, but it agrees moderately well with the Monte-Carlo calculations of Kyser (1979), Geiss and Kyser (1979) and Newbury and Myklebust (1979) and the few experimental measurements of beam broadening, Hutchings et al (1979) and Cliff and Lorimer (1981). Cliff and Lorimer (1981) have shown that the expression of Goldstein et al (1977) corresponds exactly to one limit predicted by plural scattering theory, that of a Gaussian distribution of scattering events, while the other limit, a single scattering event, predicts less broadening and coincides with Monte-Carlo calculations.

It is essential to know the electron intensity distribution in the incident probe if beam broadening in the specimen is to be calculated and the activated volume for $x$-ray microanalysis to be determined. The electron intensity distribution in the probe is often assumed to be Gaussian (Doig et al 1981), but this is often not the true situation. Figure 10 shows a microdensitometer trace of a micrograph of the analysis probe in a Philips EM400T operating in the TEM nanoprobe mode, $100 \mathrm{kV}$, eucentric position, spot size 6. A Gaussian distribution is superimposed on the experimentally measured profile. The experimental probe has a long, non-Gaussian, tail which, in the illustration, contains a significant proportion of the total current (the true three-dimensional ratio of 'tail' to 'probe' current is larger than the apparent ratio in the two-dimensional profile shown in figure 10.* The ratio of the electron intensity distribution in the 'tail' to that in the 'probe' depends on the convergence angle of the beam. A highly convergent beam, which produces the smallest possible 'probe', for a given set of operating conditions, also produces a large 'tail'. With a less convergent beam the 'tail' can be dramatically reduced, although the 'probe' diameter is increased. The origin of the 'tail'

* This distribution of electron intensity in the analysis probe is not unique to the EM400T. It is an inherent characteristic of any highly convergent probe-forming system. 


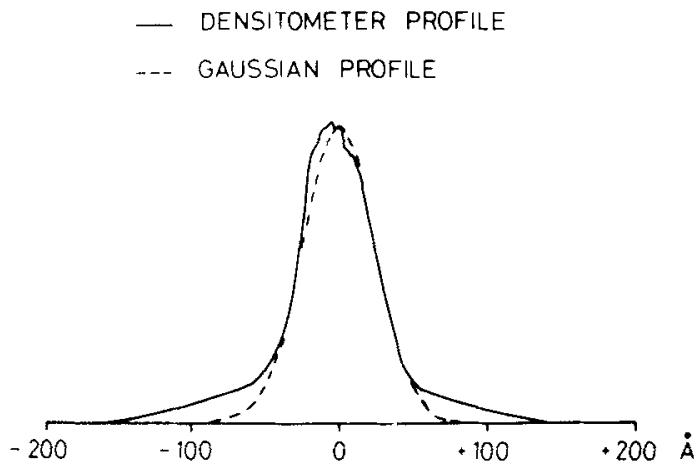

Figure 10. Microdensitometer profile of a focussed probe with a Gaussian profile superimposed. From Lorimer (1981).

is thought to be due to a combination of the high degree of illumination convergence and spherical aberration.

\section{Summary}

The physics of $\mathbf{x}$-ray production, absorption and detection is moderately wellunderstood, and it has been applied to develop a model for the quantitative $x$-ray microprobe analysis of thin specimens. The major correction to the simple 'ratio' technique is that of $\mathrm{x}$-ray absorption within the specimen, and this can be made if the sample thickness is known. With the availability of analysis probes of less than $10 \mathrm{~nm}$ in diameter, beam spreading within the specimen provides the ultimate limit to spatial resolution for chemical analysis. If 'true' concentration profiles on the nm scale are to be determined it is important to know the electron intensity distribution within the analysis probe, a distribution which may not be Gaussian.

\section{References}

Cliff G and Lorimer G W 1975 J. Microsc. 103203

Cliff G and Lorimer G W 1981 Quantitative microanalysis with high spatial resolution (eds) G W Lorimer, M Jacobs and P Doig (London: Metals Society) p. 47

Doig P, Lonsdale D and Flewitt P E J 1981 Quantitative microanalysis with high spatial resolution (eds) G W Lorimer, M Jacobs and P Doig (London: Metals Society) p. 41

Geiss R H and Kyser D 1979 Ultramicroscopy 3397

Goldstein J I, Costley J L, Lorimer G W and Reed S J B 1977 Quantitative x-ray analysis in the electron microscope, Proc.Workshop on Analytical EM (ed) O Johari (Chicago: IITRI) p. 315

Goldstein J I and Williams D B 1981 Quantitative microanalysis with high spatial resolution (eds) G W Lorimer, M Jacobs and P Doig (London: Metals Society) 5

Green M and Cosslett V E 1961 Proc. Phys. Soc. 711206

Hutchings R, Loretto M H, Jones I P and Smallman R E 1979 Ultramicroscopy 3401

Kyser D F 1979 Introduction to analytical electron microscopy (eds) J J Hren, J I Goldstein and D C Joy (New York: Plenum) p. 199

Lorimer G W, Al-Salman S A and Cliff G 1977 Developments in Electron Microscopy and Analysis (ed) D C Misell (London: Institute of Physics) 369 
Lorimer G W 1981 Developments in electron microscopy and analysis (ed) M J Goringe (London: Institute of Physics) p. 147

McGill R J and Hubbard F H 1981 Quantitative microanalysis with high spatial resolution (eds) G W Lorimer, M Jacobs and P Doig (London: Metals Society) 30

Morris P L, Davies N C and Treverten J A 1977 Developments in electron microscopy and analysis (ed) D C Misell (London: Institute of Physics) 374

Newbury D E and Myklebust 1979 Ultramicroscopy 3391

Nockolds C, Nasir M J, Cliff G and Lorimer G W 1979 Developments in electron microscopy and analysis (ed) T Mulvey (London: Institute of Physics) 417

Philibert J and Tixier R 1975 Physical aspects of electron microscopy and microbeam analysis (eds) B M Siege! and D R Beaman (New York: Wiley) 333

Ridley N and Lorimer G W 1981 Quantitative microanalysis with high spatial resolution (eds) G W Lorimer, M Jacobs and P Doig (London: Metals Society) 80

Wood J, Williams D B and Goldstein J I 1981 Quantitative microanalysis with high spatial resolution (eds) G W Lorimer, M Jacobs and P Doig (London: Metals Society) 24 